\title{
Orodental Local Drug Delivery
}

${ }^{1}$ Nandita Ahanthem, ${ }^{2}$ Sowbhagya Malligere Basavaraju, ${ }^{3}$ Balaji Pachipulusu, ${ }^{4}$ Nikhat Gazge

\begin{abstract}
Oral mucosal diseases are the most common diseases affecting humans and these can be treated with the use of various drugs. These drugs can be administered via many routes to produce its pharmacological bioeffects. One such site is the oral cavity, where both local and systemic deliveries of drug can take place. Oral route has been the most convenient and commonly employed route of drug delivery. The oral mucosa's accessibility, excellent blood supply, bypass of hepatic first pass metabolism, rapid repair, and permeability profile make it an attractive site for local and systemic drug deliveries. Local drug delivery allows topical treatment of various oral mucosal diseases, as it provides a more targeted and efficient drug-delivery option than systemic delivery. This review highlights various methods of drug delivery and important aspects of mucoadhesive drug delivery and drug dosage for treatment of orodental diseases.
\end{abstract}

Keywords: Dosage forms, Drug delivery, Mucoadhesive, Oral mucosa, Oral mucosal disease, Transmucosal.

How to cite this article: Ahanthem N, Basavaraju SM, Pachipulusu B, Gazge N. Orodental Local Drug Delivery. J Health Sci Res 2015;6(2):41-46.

\section{Source of support: Nil}

Conflict of interest: None

\section{INTRODUCTION}

The oral mucosa is the lining of the oral cavity that communicates with the exterior and covers most of the oral cavity apart from the teeth. Its main purpose is to act as a barrier and protect the deeper tissues, such as fat, muscle, and nerve and blood supplies from mechanical injuries, such as trauma during chewing; it also prevents the entry of bacteria and some toxic substances into the body. Oral mucosal diseases are among the most common diseases affecting humans, and they can be effectively treated by topical therapeutic approaches. But there are various limitations associated with these formulations that result in the short retention time of the drugs. ${ }^{1}$

\footnotetext{
${ }^{1,4}$ Postgraduate Student, ${ }^{2}$ Reader, ${ }^{3}$ Professor

${ }^{1-4}$ Department of Oral Medicine and Radiology, RajaRajeswari Dental College \& Hospital, Bengaluru, Karnataka, India

Corresponding Author: Nandita Ahanthem, Postgraduate Student, Department of Oral Medicine and Radiology RajaRajeswari Dental College \& Hospital, Bengaluru Karnataka, India, Phone: +918028437150 , e-mail: nanditaahan them24@gmail.com
}

For these reasons, novel drug dosage forms for local drug delivery should be able to overwhelm the following drawbacks: ${ }^{2}$

- Rapid loss of drug from the site of absorption by means of salivary action and mechanical stress.

- Inadequate distribution of drugs within the areas of the oral cavity.

- Patient discomfort due to unpleasant taste sensations.

- Barrier effect of oral mucosa.

\section{Structure of Oral Mucosa and its Permeability facilitating Drug Delivery}

The oral mucosa is composed of lamina propria followed by the submucosa as the innermost layer covered by the outermost layer of stratified squamous epithelium. It has a total surface area of about $200 \mathrm{~cm}^{2}$, consisting of two anatomical and functional layers, i.e., a thick stratified squamous avascular epithelium and an underlying avascular layer of mesodermal origin. ${ }^{1}$ The epithelium is similar to stratified squamous epithelia found in the rest of the body, in that it has a mitotically active basal cell layer advancing through a number of differentiating intermediate layers to the superficial layers, where cells are shed from the surface of the epithelium. ${ }^{3,4}$ The epithelium of the buccal mucosa is about 40 to 50 cell layers thick, while that of the sublingual epithelium contains somewhat fewer layers. ${ }^{5}$ Thicknesses depending on the site are as given in Table 1.

The permeability of the buccal mucosa is 4 to 4000 times greater than that of the skin. ${ }^{6}$ This permeability feature of the oral mucosa is the most important factor that determines the appropriate drug formulations so that the drug gets absorbed and reaches the deeper layers of the oral mucosa. The permeability of oral mucosa is attributed to intercellular materials derived from membrane-coating granules, which are found in the intermediate cell layers of both keratinized and nonkeratinized epithelia. ${ }^{5}$ The components of the membrane-coating granules differ from keratinized and nonkeratinized

Table 1: Thickness and permeability of oral mucosa

\begin{tabular}{llll}
\hline Tissue & Structure & Thickness $(\mu \mathrm{m})$ & Permeability \\
\hline Buccal & NK & $500-600$ & Intermediate \\
Sublingual & NK & $100-200$ & Very good \\
Gingival & K & 200 & Poor \\
Palatal & K & 250 & Poor \\
\hline
\end{tabular}

NK: Nonkeratinized; K: Keratinized 
epithelia. The keratinized epithelia are composed of lamellar lipid stacks, which include sphingomyelin, ceramides, and nonpolar lipid, whereas keratinized epithelia contains nonlamellar lipid, i.e., cholesterol ester and glycosphingolipids. ${ }^{6}$ The permeability of the oral mucosae decreases in the order of sublingual, buccal, and palatal. This ranking is based on the relative thickness and degree of keratinization of these tissues, where the sublingual mucosa is relatively thin and nonkeratinized, the buccal mucosa is thicker and nonkeratinized, and lastly, the palatal mucosa is intermediate in thickness but keratinized. ${ }^{4}$

\section{PRINCIPLES OF DRUG ABSORPTION VIA THE ORAL MUCOSA}

The surface area of the oral mucosa is relatively small $\left(200 \mathrm{~cm}^{2}\right)$ compared with the gastrointestinal tract $\left(350,000 \mathrm{~cm}^{2}\right)$ and skin $\left(20,000 \mathrm{~cm}^{2}\right){ }^{7,8}$ The oral mucosa is highly vascularized, and therefore, any drug diffusing into the oral mucosa membranes has direct access to the systemic circulation via capillaries and venous drainage, bypassing the gastrointestinal tract and first pass metabolism in the liver. The rate of blood flow through the oral mucosa is substantial and is generally not considered to be the rate-limiting factor in the absorption of drugs by this route. ${ }^{3}$ For a drug to pass through the oral mucosa, it must first diffuse through the lipophilic cell membrane and then pass through the hydrophilic cells of the oral epithelium. Thus, the oral mucosa provides both hydrophilic and hydrophobic barriers that must be overcome for efficient mucosal delivery. ${ }^{5}$

\section{ROUTES OF DRUG TRANSPORT VIA ORAL MUCOSA}

Compounds or molecules with different chemical properties penetrate the barrier region of the oral mucosa via different routes. There are two permeation pathways for passive drug transport across the oral mucosa, i.e., paracellular and transcellular routes (Fig. 1). ${ }^{9}$ Permeants can use these two routes simultaneously, but one route is usually preferred over the other depending on the physicochemical properties of the diffusant. Delivery of

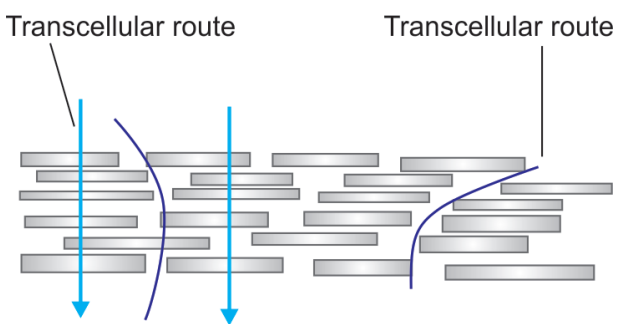

Fig. 1: Permeation pathways for passive drug transport across the oral mucosa ${ }^{9}$ drugs via the membranes of the oral cavity is classified into three categories: Buccal delivery, sublingual delivery, and local delivery. ${ }^{10,11}$

\section{LOCAL ORAL DRUG DELIVERY}

Drug delivery via the oral mucosa can be subdivided into two different approaches:

(i) Drug delivery via keratinized mucosa.

(ii) Drug delivery via nonkeratinized mucosa (Flow Chart 1).

The selection of one path depends on regional differences in terms of anatomic and permeability features, which exist between these oral mucosal sites. The keratinized mucosa, i.e., gingiva and hard palatal mucosa, is still not considered a valid site for the systemic administration of drugs, and they should be considered as useful sites for local drug delivery only in treating oral diseases localized at the gingiva or palate. The rationale behind gingival drug delivery is that concentrated amounts of active drugs can be delivered to the precise site of the disease process with a minimal systemic uptake of the medication. Such devices could be useful adjuncts to conventional mechanical therapy, and they are associated with low side effects and drug interactions. Drug delivery via the nonkeratinized mucosa can be subdivided into two approaches:

(i) Sublingual drug delivery (across the mucosa lining the floor of mouth).

(ii) Buccal drug delivery (mainly via the buccal mucosa lining the cheeks, including systemic and/or local delivery).

The sublingual mucosa is more permeable and thinner than the buccal mucosa, making it a feasible site if a rapid onset is desired and in treating acute disorders.

The buccal mucosa is considerably less permeable than the sublingual mucosa, and it is unable to provide the rapid onset of absorption observed with sublingual administration. Hence, buccal mucosa constitutes a preferred route for the systemic treatment of chronic disorders when the sustained delivery of systemically acting drugs is required, thereby overcoming the drawbacks when they are administered by conventional routes. ${ }^{12,13}$

Flow Chart 1: Drug delivery via oral mucosa

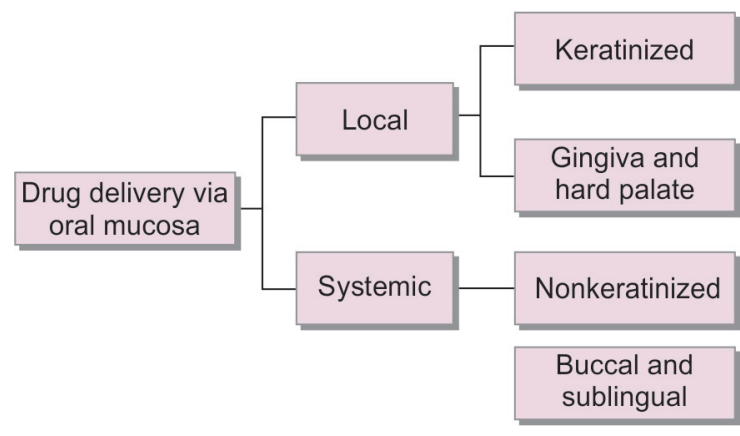




\section{Oral Local Drug}

Delivery consists of a more efficient drug-delivery approach than systemic delivery for the treatment of oral conditions. Many oral diseases are chronic and require chronic treatment regimens. In addition, most of the oral diseases can be treated locally, without the need for ingestion and the systemic distribution of drugs. Thus, local drug delivery provides a more targeted delivery, as smaller amounts of drug can be easily targeted at the site of the disease, thereby reducing side effects. ${ }^{4}$ Furthermore, undesirable taste sensations, especially during long-term treatment, could restrict the value of this route of administration.

\section{Advantages}

Rapid onset of action and absorption, increased bioavailability, decreased drug dose, self-administration, and reduced systemic toxicity.

\section{Disadvantages}

Taste factor, dislodgement of delivery device, permeability barraier, washing away of drug by saliva, and highly enzymatic environment.

\section{Pharmaceutical Consideration and Formulation of Local Drug Delivery}

Drug selection for oral transmucosal delivery is limited by the physicochemical properties of the drugs themselves. Drugs must have unique physicochemical properties (proper balance between solubility and lipophilicity) to be delivered transmucosally. Only a few milligrams of drug can cross the oral mucosa, even if the drug has a favorable profile for oral mucosal delivery. Factors influencing drug release are an important consideration; ideal formulation and its degradation products should be nontoxic, nonirritant, and free from leachable impurities, and lastly, they should not aid in development of secondary infections. An ideal transmucosal drug-delivery system must meet several prerequisites. Firstly, it should rapidly attach to the mucosal surface and maintain a strong interaction to prevent displacement and spontaneous adhesion of the system at the target site, and it can be achieved through bioadhesion promoters that use tethered polymers. Contact time should also be sufficiently long at the target site, normally longer than that needed for complete drug release. Secondly, bioadhesion performance should not be impacted by the surrounding environmental $\mathrm{pH}$. Other considerations include high drug loading, complete drug release, and convenient administration. ${ }^{14}$

\section{METHOD OF ORAL LOCAL DRUG DELIVERY}

\section{Mucoadhesive Dosage Forms}

Mucoadhesive dosage forms are a new type of formulation design. It was first introduced in the early 1980s. ${ }^{15}$ Mucoadhesion is a complex phenomenon; the first step is the spreading, wetting, and dissolution of mucoadhesive polymer at the interface and the second step is the mechanical or physical entanglement between the polymer and the tissue surface mucus layer, resulting in an interpenetration layer. The next step is the result of chemical interactions, such as covalent and ionic bonds, hydrogen bonding, and Van der Waals' interactions. Hydrogen bonds and hydrophobic interactions are the most desirable in developing mucoadhesive systems as strong primary bonds (e.g., covalent bonds and ionic bonds). In addition, the density of the cross-linking agent significantly affects mucoadhesion. Mucoadhesive polymers and novel copolymers are used to enhance the intrinsic mucoadhesive properties. ${ }^{16,17}$

According to the mechanism by which a drug is released from the delivery device, dosage forms can be classified as (i) a monolithic (or "matrix") type (ii) and a reservoir (or "membrane-controlled") type. In the former, the drug is uniformly dispersed or dissolved in the polymer matrix and drug release is effected by diffusion through the polymer network. In the latter, a drug reservoir is entrapped between an impermeable backing layer and a polymeric membrane that controls the rate of drug release. ${ }^{1}$

Mucoadhesive systems for oral local drug delivery include adhesive tablets, adhesive patches, adhesive films or pellicles, adhesive semisolid systems (gels, ointments) and adhesive liquid systems (sprays, mouthwashes), chewing gum, hydrogels, hollow fibers, and microspheres.

\section{Solid Forms (Tablets and Lozenges)}

Although these formulations vary in shape and size, they share many common characteristics. This method of delivery is simple for patients to use. The solid formulations dissolve in the oral cavity; later on, it is released and exposed to the entire mucosa as well as on the top third layer of the esophageal mucosa. Buccal tablets are small, flat, and oval with an approximate diameter of 5 to $8 \mathrm{~mm}$ and thickness of about $2 \mathrm{~mm} \cdot{ }^{18}$ In the presence of saliva, they adhere to the mucosal surface until dissolution and/or drug release is complete. To prevent drug loss from the top surface, specialized tablets with two layers have been developed; also, they contain a drugloaded bioadhesive layer and an impermeable backing layer to promote unidirectional drug absorption and 
to minimize drug leakage in the oral cavity. The other surfaces of these bioadhesive tablets are coated with water-impermeable hydrophobic substances (e.g., ethyl cellulose or oil). Bilayered adhesive tablets have been designed. This type of dosage form can be used only for the treatment of localized oral lesions because its main disadvantage is the lack of the physical flexibility of the material applied to the mucosa. ${ }^{19,20}$

\section{Adhesive Patches and Films}

Flexible adhesive patches and films have been developed to overcome the drawbacks of other dosage forms that possess unique characteristics, including relatively rapid onset of drug delivery, sustained drug release, and rapid decline in the serum drug concentration when the patch is removed. Oral mucosal patches can be classified as (i) patches with a dissolvable matrix, (ii) patches with a nondissolvable backing, and (iii) patches with a dissolvable backing. Patches with a dissolvable matrix are designed to release drug into the oral cavity. Oral patches and films have high flexibility, thus facilitating a long residence/retention time, provide a more accurate dosing of drug delivery as compared with other dosage forms (gels and sprays), and protect the underlying diseased tissues, thus reducing pain and increasing treatment effectiveness. They are useful in the treatment of mild or severe diffuse oral diseases, particularly in chronic oral diseases where long-term drug regimens are often required. ${ }^{4}$

\section{Adhesive Semisolid Systems (Gels, Ointments)}

These modalities have the advantage of easy dispersion throughout the oral mucosa. They form an intimate contact with the mucosal membrane and rapidly release drugs at the absorption site. This delivery system may not be accurate as compared with tablets, patches, or films. There is poor retention time of the gels at the site of application because body fluids, such as saliva will quickly wash them away from the site of action. For these reasons, they are of limited use for drugs with a narrow therapeutic window. ${ }^{21,22}$ A major application of adhesive gels could be the local delivery of medicinal agents for the treatment of periodontitis, recurrent aphthous stomatitis, traumatic ulcers, radiation- or chemotherapy-induced oral mucositis, chronic immunologically mediated oral lesions, hyposalivation, and healing of wounds.

\section{Adhesive Liquid Systems (Oral Rinse and Sprays)}

These systems produce a very fine mist that tends to coat the entire oral mucosa, thereby increasing the total surface area through which drug molecules can be absorbed, and compositions possessing high mucoadhesion and viscoelasticity. Bioadhesive liquid systems have been proposed for the treatment of several oral diseases, such as, oral lichen planus and other immunologically mediated diseases, aphthous stomatitis, oral mucositis, hyposalivation, and potentially malignant disorders, such as, leukoplakia and erythroplakia. ${ }^{23}$ An ideal adhesive spray system should be able to produce spray patterns of a suitable ovality and particle size. The ovality of the spray pattern refers to the symmetric oval shape of spray particles, and it is believed that the more the oval shape of spray particles the greater will be the ability of the particles to cover the whole mucosa. ${ }^{24}$

\section{Vesicular System}

\section{Multiparticulates, Microparticles, and Nanoparticles}

Oral delivery systems based on multiparticulates, microparticles, and nanoparticles exhibit improved performance in comparison with monolithic matrix tablets. It is an effort to develop an effective bioadhesive system. Nanoparticle formation ensures even distribution of the drug adhered well to the mucosa, leading to good absorption. Also, liposomes have been used in local drug delivery to oral mucosa. ${ }^{25,26}$

\section{LOCAL DRUG DELIVERY VS SYSTEMIC DRUG DELIVERY}

Local drug delivery can provide a more targeted and efficient drug-delivery option than systemic delivery for diseases of the oral mucosa.

Systemic delivery required higher drug dosage, peak level of a few hours in plasma, and more side effects (Table 2). ${ }^{27-44}$

Local delivery needs lower dosage, peak level within a few minutes, and reduced side effects. ${ }^{23}$

\section{CONCLUSION}

Oral transmucosal technology offers an alternative means of administering drugs, which allows more rapid absorption into the bloodstream. This method is noninvasive, convenient for patients, and provides a more targeted therapeutic option, thereby reducing drug dose and systemic toxicity. An important outcome from this novel approach could be the possibility of providing more effective treatment regimens to a wider range of patients suffering from severe or refractory oral diseases. There are several limitations, such as irritancy, taste factor, and retention at the site of application, which need to be considered in the design of such medicines. Hence, considering the potential and specific advantages of oral transmucosal drug delivery, it is preferred over systemic routes of delivery. 
Table 2: Common oral mucosal lesion and suitable formulations

\begin{tabular}{|c|c|c|}
\hline Drug & Form & Results \\
\hline \multicolumn{3}{|l|}{ Oral lichen planus } \\
\hline Clobetasol $^{27}$ & Mucoadhesive gel & Contain $24 \mu \mathrm{g}$, TDS appeared to be effective avoiding side effect \\
\hline Cyclosporine and Tacrolimus 28,29 & $\begin{array}{l}\text { Mucoadhesive gel } \\
\text { and oral rinse }\end{array}$ & Symptomatic relief \\
\hline \multicolumn{3}{|l|}{ Oral mucositis } \\
\hline TGF- $\beta 3^{30}$ & Oral rinse & $\begin{array}{l}\text { Inhibits epithelial proliferation, penetrates the epithelium, and } \\
\text { is detected in the basal cell layer at therapeutically effective } \\
\text { concentration }\end{array}$ \\
\hline TGF- $\beta 3^{31}$ & Chitosan gel & Improved drug retention, protection against Candida infection \\
\hline Gengigel, MuGuard ${ }^{14}$ & $\begin{array}{l}\text { Mucoadhesive covering } \\
\text { agents }\end{array}$ & $\begin{array}{l}\text { Physical coating, protection for thinned or ulcerated mucosa } \\
\text { symptomatic relief }\end{array}$ \\
\hline \multicolumn{3}{|l|}{$\begin{array}{l}\text { Potentially malignant disorder } \\
\text { and oral cancer }\end{array}$} \\
\hline $5-\mathrm{FU}^{32}$ & Matrix tablet & $5 \% 5$-FU useful in OSCC treatment \\
\hline Tretinoin 33 & Patch & Chemoprevention \\
\hline 5-aminolevulinic acid ${ }^{34}$ & Gel & Followed by photodynamic therapy, complete response was obtained \\
\hline Idarubicin ${ }^{35}$ & Solid lipid nanoparticle & Provides higher intracellular level relative to bolus administration \\
\hline \multicolumn{3}{|l|}{ Recurrent apthous ulcer } \\
\hline Amelexanon ${ }^{36}$ & Mucoadhesive tablet & Symptomatic relief and reduction in size of ulcer \\
\hline Hydroxyapatite $^{37}$ & Mucoadhesive gel & Symptomatic relief, reduction in size and number \\
\hline \multicolumn{3}{|l|}{ Xerostomia } \\
\hline Interferon alpha ${ }^{38}$ & Tablets & TDS, enhances salivary secretion \\
\hline Physostigmine ${ }^{39}$ & Gel (1.8 mg) & Relief in feeling dryness \\
\hline \multicolumn{3}{|l|}{ Oral diseases } \\
\hline Minocycline ${ }^{40}$ & Gels/microsphere & Improve attachment level and probing depth \\
\hline Doxyxlycline $e^{41}$ & Gel & \\
\hline Tetracycline $e^{42}$ & Mucoadhesive patch & $\begin{array}{l}\text { Tetracycline with carvasol combination - effective against bacterial } \\
\text { infection and candidiasis }\end{array}$ \\
\hline Metronidazole ${ }^{43}$ & Mucoadhesive tablets & Sustained released - periodontitis \\
\hline Miconazole ${ }^{44}$ & Buccal tablet & Fungal infection \\
\hline Clotrimazole $^{44}$ & Troche/cream/gel & Symptomatic relief \\
\hline
\end{tabular}

TGF-ß3: Transforming growth factor beta-3; 5-FU: 5-fluorouracil; OSCC: Oral squamous cell carcinoma; TDS: Three times a day

\section{REFERENCES}

1. Paderni C, Compilato D, Giannola LI, Campisi G. Oral local drug delivery and new perspectives in oral drug formulation. Oral Surg Oral Med Oral Path Oral Radiol 2012 Sep;114(3):25-34.

2. Mathew AP. Oral local drug delivery: an overview. Pharm Pharmacol Res 2015 Jul;3(1):1-6.

3. Madhav NVS, Semwal R, Semwal DK, Semwal RB. Recent trends in oral transmucosal drug delivery systems: An emphasis on the soft palate route. Exp Opin Drug Deliv 2012 Jun;9(6):629-645.

4. Madhav NVS, Shakya AK, Shakya P, Singh K. Orotransmucosal drug delivery systems: A review. J Control Release 2009 Nov 16;140(1):2-11.

5. Munot NM, Gujar KN. Orodental delivery systems: an overview. Int J Pharm Pharm Sci 2013 May;5(3):74-83.

6. Lee J, Kil S, Choi YW. The effect of the storage conditions on permeability of porcne buccal mucosa. Arch Pharm Res 2002 Aug;25(4):546-549.

7. Dawes C. Glandsize estimation and body mass index improve salivary flow rate assessment. Arch Oral Biol 2007 May;52(5):409-410.

8. Squier CA, Wertz PW. Permeability and the pathophysiology of oral mucosa. Adv Drug Deliv Rev 1993;12:13-24.

9. Rathbone M, Drummond B, Tucker I. Oral cavity as a site for systemic drug delivery. Adv Drug Del Rev 1994;13:1-10.
10. Chinna K. Local drug delivery: A review. Indian J Dental Sci 2012;4:66-69.

11. Nibha KP, Pancholi SS. An overview on sublingual route for systemic drug delivery. Int J Res Pharmaceut Biomed Sci 2012 Jun;3(2):913-923.

12. Harris D, Robinson JR. Drug delivery via the mucous membranes of the oral cavity. J Pharmacol Sci 1992 Jan;81(1):1-10.

13. Hearnden V, Sankar V, Hull K, Juras DV, Greenberg M, Kerr AR, Lockhart PB, Patton LL, Porter S, Thornhill MH. New developments and opportunities in oral mucosal drug delivery for local and systemic disease. Adv Drug Deliv Rev 2012 Jan;64(1):16-28.

14. Sudhakar Y, Kuotsu K, Bandyopadhyay AK. Buccal bioadhesive drug delivery - a promising option for orally less efficient drugs. J Control Release 2006 Aug 10;114(1):15-40.

15. Ishida M, Machida $Y$, Nambu N, Nagai T. New mucosal dosage form of insulin. Chem Pharm Bull (Tokyo) 1981 Mar;29(3):810-816.

16. Tur K, Ch'ng H. Evaluation of possible mechanisms. Int J Pharm 1998;160:61-74.

17. Park K, Robinson J. Bioadhesive polymers as platforms for oral-controlled drug delivery: Method to study bioadhesion. Int J Pharm 1984;19:107-127.

18. Mizrahi B, Domb AJ. Mucoadhesive polymers for delivery of drugs to the oral cavity. Recent Pat Drug Deliv Formul 2008;2(2):108-119. 
19. Ceschel GC, Bergamante V, Calabrese V, Biserni S, Ronchi C, Fini A. Design and evaluation in vitro of controlled release mucoadhesive tablets containing chlorhexidine. Drug Dev Ind Pharm 2006 Jan;32(1):53-61.

20. Llabot JM, Manzo RH, Allemandi DA. Double-layered mucoadhesive tablets containing nystatin. AAPS PharmSciTech 2002 Sep;3(3):E22.

21. Avinash N. Science and technology of bioadhesive-based targeted oral delivery systems. Pharma Technol 2008 Nov;32(11):100-121.

22. Needleman IG, Martin GP, Smales FC. Characterisation of bioadhesives for periodontal and oral mucosal drug delivery. J Clin Periodontol 1998 Jan;25(1):74-82.

23. Sankar V, Hearnden V, Hull K, Juras DV, Greenberg MS, Keer AR, Lockhart PB, Patton LL, Porter S, Thornhill MH. Local drug delivery for oral mucosal diseases challenges and opportunities. Oral Dis 2011 Apr;17(1):73-82.

24. Hiremath PS, Bhonsle SA, Thumma S, Vemulapalli V. Recent patents on oral combination drug delivery and formulations. Recent Pat Drug Deliv Formul 2011 Jan;5(1):52-60.

25. Salmaso S. Muco-adhesive multivesicular liposomes as an effective carrier for transmucosal insulin delivery. J Drug Target 2007 Jul;15(6):417-427.

26. Velpula N. Oral mucosal drug delivery - an adjunct to the current therapeutic strategies in the dental management of oral diseases: review. Oral Health Dent Manag 2014 Dec;13(4):1034-1040.

27. Cilurzo F, Gennari CG, Selmin F, Epstein JB, Gaeta GM, Colella G, Minghetti P. A new mucoadhesive dosage form for the management of oral lichen planus: formulation study and clinical study. Eur J Pharm Biopharm 2010 Nov;76(3):437-442.

28. Conrotto D, Carbone M, Carrozzo M, Arduino P, Broccoletti R, Pentenero M, Gandolfo S. Cyclosporin vs. clobetasol in the topical management of atrophic and erosive oral lichen planus: A double-blind, randomized controlled trial. Br J Dermatol 2006 Jan;154(1):139-145.

29. Rouxel AM, Toux G, Misery L. Tacrolimus mouthwash as second-line treatment for erosive oral lichen planus. Ann Dermatol Venereol 2010 Oct;137(10):648-649.

30. Sonis ST, Van Vugt AG, Brien JP, Muska AD, Bruskin AM, Rose A, Haleu JD. Transforming growth factor-beta 3 mediated modulation of cell cycling and attenuation of 5-fluorouracil induced oral mucositis. Oral Oncol 1997 Jan;33(1):47-54.

31. Squier CA, Kremer MJ, Bruskin A, Rose A, Haley JD. Oral mucosal permeability and stability of transforming growth factor beta-3 in vitro. Pharmacol Res 1999 Oct;16(10):1557-1563.

32. Gaeta GM, Gombos F, Femiano F, Battista C, Minghetti P, Montanari L, Satriano RA, Argeziano G. Acitretin and treatment of the oral leucoplakias. A model to have an active molecules release. J Eur Acad Dermatol Venereol 2000 Nov;14(6):473-478.

33. Mulshine JL, Atkinson JC, Greer RO, Papadimitrakopoulou VA, Van Waes C, Rudy S, Martin JW, Steinberg SM, Leiwehr DJ, Avis I. Randomized, double-blind, placebo controlled phase IIb trial of the cyclooxygenase inhibitor ketorolac as an oral rinse in oropharyngeal leukoplakia. Clin Cancer Res 2004 Mar 1;10(5):1565-1573.

34. Sieron' A, Namysłowski G, Misiołek M, Adamek M, KawczykKrupka A. Clinical response to photodynamic therapy in premalignant lesions and advanced head and neck carcinomas. Otolaryngol Pol 2003 Oct; 57(4):501-504.

35. Holpuch AS, Hummel GJ, Tong M, Seghi GA, Pei P, Ma P, Mumper RJ, Mallery SR. Nanoparticles for local drug delivery to the oral mucosa: proof of principle studies. Pharmacol Res 2011 Jul;27(7):1224-1236.

36. Liu J, Zeng X, Chen Q, Cai Y, Chen F, Wang Y, Zhou H, Lin M, Shi J, Wang Z, et al. An evaluation on the efficacy and safety of amlexanox oral adhesive tablets in the treatment of recurrent minor aphthous ulceration in a Chinese cohort: a randomized, double-blind, vehicle-controlled, unparallel multicenter clinical trial. Oral Surg Oral Med Oral Pathol Oral Radiol Endod 2006 Oct;102(4):475-481.

37. Lee JH, Jung JY, Bang D. The efficacy of topical $0.2 \%$ hyaluronic acid gel on recurrent oral ulcers: comparison between recurrent aphthous ulcers and the oral ulcers of Behcet's disease. J Eur Acad Dermatol Venereol 2008 May;22(5): 590-595.

38. Khosravani N, Ekström J, Birkhed D. Intraoral stimulation of salivary secretion with the cholinesterase inhibitor physostigmine as a mouth spray: a pilot study in healthy volunteers. Arch Oral Biol 2007 Nov;52(11):1097-1101.

39. von Bultzingslowen I, Sollecito TP, Fox PC, Daniels T, Jonsson R, Lockhart PB, Wray D, Brennan MT, Carrozzo M, Gandera B, et al. Salivary dysfunction associated with systemic diseases: systematic review and clinical management recommendations. Oral Surg Oral Med Oral Pathol Oral Radiol Endod 2007 Mar;103(Suppl):1-15.

40. Vinholis AH, Figueiredo LC, Marcantonio Júnior E, Marcantonio RA, Salvador SL, Goissis G. Subgingival utilization of a $1 \%$ chlorhexidine collagen gel for the treatment of periodontal pockets. A clinical and microbiological study. Braz Dent J 2001;12(3):209-213.

41. Renvert S, Lessem J, Dahlén G, Renvert H, Lindahl C. Mechanical and repeated antimicrobial therapy using a local drug delivery system in the treatment of peri-implantitis: a randomized clinical trial. J Periodontol 2008 May;79(5): 836-844.

42. Obaidat RM, Bader A, Al-Rajab W, Abu Sheikha G, Obaidat A. A preparation of mucoadhesive oral patches containing tetracycline hydrochloride and carvacrol for treatment of local mouth bacterial infections and candidiasis. Sci Pharm 2011 Mar;79(1):197-212.

43. Perioli L, Ambrogi V, Rubini D, Giovagnoli S, Ricci M, Blasi $P$, Rossi C. Novel mucoadhesive buccal formulation containing metronidazole for the treatment of periodontal disease. J Control Release 2004 Mar 24;95(3):521-533.

44. Dangi YS, Soni ML, Namdeo KP. Oral candidiasis: a review. Int J Pharm Sci 2010;2(3):36-41. 